\title{
Healthy lifestyle consultation based on traditional Chinese medicine versus routine patient education in the treatment of idiopathic sudden sensorineural hearing loss after failure of systemic therapy: study protocol for a clinical randomised trial
}

\author{
Ying-ping Fei ${ }^{1} \mathbb{D}$, Yun Zheng ${ }^{1 *}$, Dan Lai ${ }^{2}$, Ping Zhong ${ }^{1}$, Jing-zhe Lu' ${ }^{1}$ Gang Li and Peng $\mathrm{Liu}^{3}$
}

\begin{abstract}
Background: Idiopathic sudden sensorineural hearing loss (ISSNHL) is a major cause of deafness. Despite the advances in systemic therapy, some cases of ISSNHL are untreated, because the exact ISSNHL aetiology is unclear. Traditional Chinese medicine (TCM) has been used to treat diseases for thousands of years and is popular and widely practiced in Asia. TCM includes guidance on a healthy lifestyle. In recent decades, the relationship between lifestyle and disease has been emphasised; an unhealthy lifestyle may lead to illnesses. Thus, this study aims to compare the efficacy of lifestyle modification based on TCM with the usual consultation of ISSNHL after failure of a 2-week systemic therapy to provide a scientific basis for clinical decisions.

Methods: This study is a clinical randomised trial that involves 56 patients diagnosed with ISSNHL but who have had incomplete recovery after initial management (at least 2 weeks of routine Western medical treatment). The study is performed in accordance with the sudden hearing loss clinical guideline of the American Academy of Otolaryngology-Head and Neck Surgery, published in 2012. Participants are randomly distributed into two groups: the healthy lifestyle modification group based on TCM and the control group (1:1 ratio). Patient follow-up lasts for 3 months. The primary outcome measure is the effective rate of hearing improvement, which is defined as the proportion of patients with at least $15 \mathrm{~dB}$ of improvement in the average thresholds of hearing loss frequency. The secondary outcome measures are improvements in word recognition score, Tinnitus Handicap Inventory and visual analogue scale for ear blockage and dizziness. Assessments are made at baseline and after lifestyle modification for 1 and 3 months.
\end{abstract}

Discussion: The efficacy of healthy lifestyle modification based on a TCM programme for patients with ISSNHL with incomplete recovery after failure of initial systemic therapy is determined in this trial. Positive results will provide clinical evidence on the effects of a TCM-based healthy lifestyle, which could be recommended as salvage therapy for patients with ISSNHL.

Trial registration: Chinese Clinical Trial Registry, ChiCTR-INR-17011459. Registered on 22 May 2017.

\footnotetext{
* Correspondence: 1141679315@qq.com

${ }^{1}$ Hearing Center/Hearing and Speech Science Laboratory, Department of Otolaryngology Head and Neck Surgery, West China Hospital of Sichuan University, Chengdu, People's Republic of China

Full list of author information is available at the end of the article
}

(c) The Author(s). 2019 Open Access This article is distributed under the terms of the Creative Commons Attribution 4.0 International License (http://creativecommons.org/licenses/by/4.0/), which permits unrestricted use, distribution, and reproduction in any medium, provided you give appropriate credit to the original author(s) and the source, provide a link to the Creative Commons license, and indicate if changes were made. The Creative Commons Public Domain Dedication waiver (http://creativecommons.org/publicdomain/zero/1.0/) applies to the data made available in this article, unless otherwise stated. 


\section{Background}

Idiopathic sudden sensorineural hearing loss (ISSNHL) is a common otologic emergency that presents mostly as an acute hearing loss with an abrupt occurrence. ISSNHL is a hearing loss of more than $30 \mathrm{~dB}$ that occurs in at least three consecutive frequencies within $72 \mathrm{~h}$ [1]. Aside from hearing impairment, ISSNHL can be associated with dizziness, tinnitus and/or ear fullness/blockage. The ISSNHL incidence is approximately $5-30 / 100,000 /$ year in developed countries, such as the USA, Sweden and Japan, as revealed by national surveys [1-4]. Detailed investigations suggested that only approximately $10 \%$ of patients with ISSNHL show a specific cause [5]. Several pathophysiological mechanisms, including microcirculation, autoimmune pathology, viral infection, intracochlear membrane rupture or haematologic problems, have been proposed despite the unidentified precise cause of ISSNHL [6]. ISSNHL may not be due to a single pathological change but to a spectrum of pathologies affecting the cochlea [7].

The most common treatment option for ISSNHL is administration of corticosteroids within the first 2 weeks [4]. A total of $49-89 \%$ of patients with ISSNHL will show recovery via systemic steroid therapy, whereas therapy displays no effects on other patients [8]. Spontaneous recovery occurs in $32-65 \%$ of the cases, usually within the first 14 days $[9,10]$. However, recovery amongst patients who do not show improvement after 2 weeks is low [11]. Intratympanic steroid perfusion described in the US guidelines has been recommended as salvage therapy [1]. However, its clinical evidence remains controversial, and no existing consensus suggests the efficacy of intratympanic steroid therapy for ISSNHL $[1,12,13]$. Therefore, the failure of a 2-week treatment amongst patients with ISSNHL should be further studied, and alternate therapies should be developed.

Traditional Chinese medicine (TCM) originates from ancient China and has been used in therapeutic approaches in East Asia for more than 2500 years. TCM includes well-known herbal medicine acupuncture, massage (tui na) and lifestyle modifications, such as dietary therapy and exercise (taiji and qigong). TCM originated from Huang Di Nei Jing, a famous work of ancient TCM literature, which introduced the maintenance of the Yin-Yang balance of the internal organs by following a healthy lifestyle. From the TCM perspective, all diseases originate from a broken balance. In China, Chinese patients prefer using TCM methods with complementary and alternative medicines for disease treatment. Lifestyle change guided by TCM is also acceptable amongst Chinese people with diseases.

Lifestyle change has been suggested for patients with otological diseases whose conditions are not controlled well by medicine. Dietary habits, such as a low sodium diet, can alter the inner ear's fluid homeostasis and auditory function. The endolymph compartment maintains a low sodium concentration, and ionic balance is maintained in the surrounding perilymph and serum [14]. Evidence shows that more than $85 \%$ of patients with Meniere's disease are helped by lifestyle changes along with either medical treatment or surgical procedures. Lifestyle changes include reducing the consumption of salt, caffeinated products, chocolate and alcohol [15]. A cross-sectional study indicates the relationship between benign paroxysmal positional vertigo and inadequate carbohydrate and fibre intake and a diet rich in polyunsaturated fatty acids. Food readjustment is suggested for patients with this condition [16]. Furthermore, a descriptive longitudinal cohort study amongst 159 adult patients with chronic primary tinnitus and sleep problems has shown that TCM-based lifestyle counselling may relieve chronic primary tinnitus. After 6-26 months of follow-up, sleep quality and tinnitus loudness improved, and the effects of tinnitus on sleep, concentration and emotional state were also alleviated [17].

Therefore, this randomised controlled clinical trial was designed to evaluate the efficacy of TCM-based lifestyle modification as a salvage therapy for patients who have not recovered 14 days after the onset of ISSNHL through the use of systemic steroid therapy.

\section{Methods/design \\ Objective}

Given the significant spontaneous recovery rate and existing standard therapy for ISSNHL by using systemic glucocorticoids, patients are enrolled in the study only if no or insufficient recovery of hearing threshold is observed after the initial 14 days of systemic therapy. In addition, patients are excluded if they are reluctant to continue receiving salvage therapy. This randomised controlled trial thus evaluates the effectiveness of healthy lifestyle treatment based on TCM therapy for patients with ISSNHL who have no or insufficient recovery of hearing threshold after the initial systemic therapy for 14 days and are reluctant to continue receiving salvage therapy.

\section{Study design}

Fifty-six patients are recruited for the trial. Participants who meet the inclusion criteria and submit written informed consent are enrolled in the trial, which lasts for 3 months. Figure 1 shows the trial procedure flowchart.

This trial is registered in the Chinese Clinical Trial Registry (registration number ChiCTR-INR-17011459) and has been approved by the Biomedical Branch of the Ethics Committee of the West China Hospital of Sichuan University (identifier 2016-180). The study is performed according to the Declaration of Helsinki 


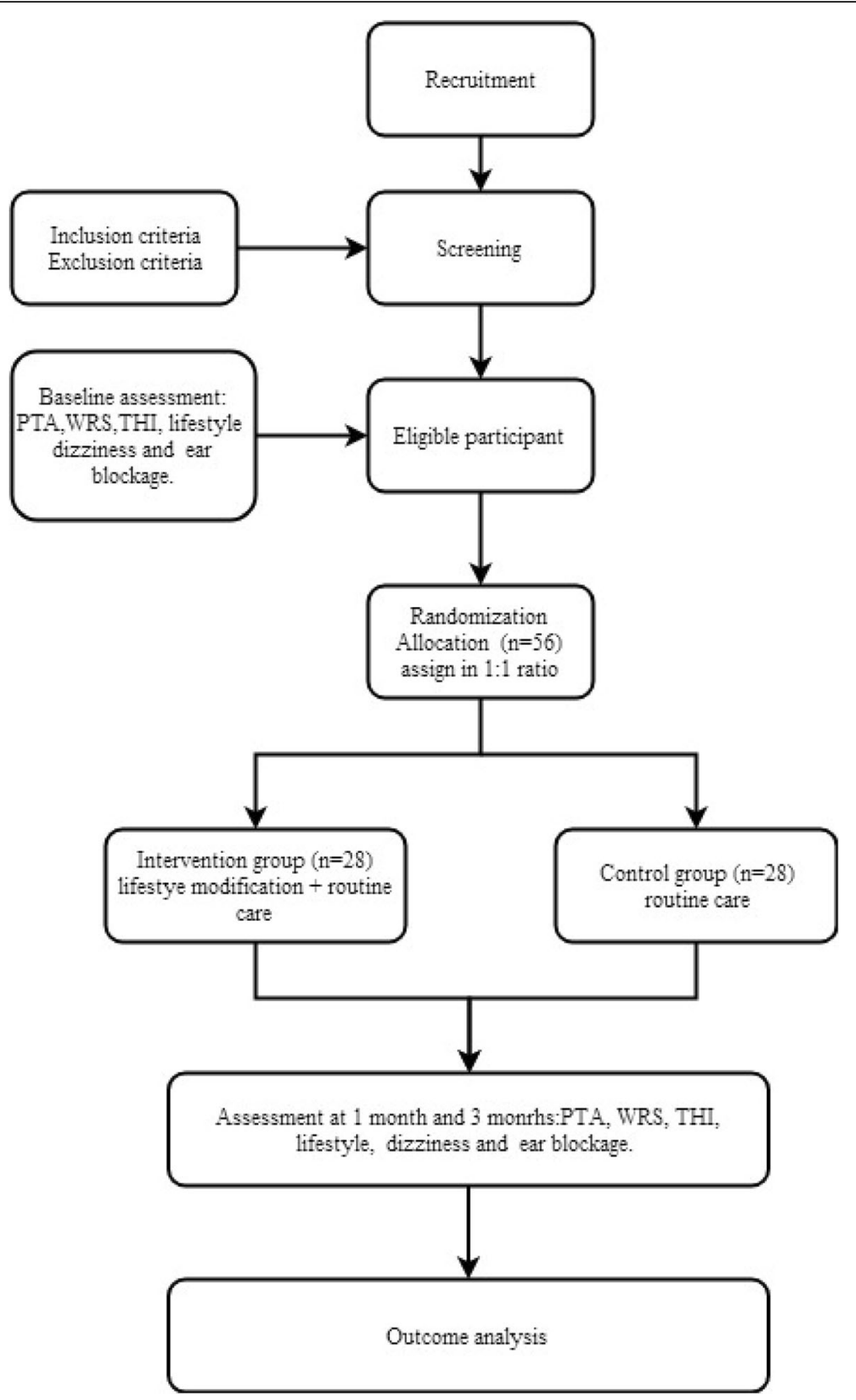

Fig. 1 Trial flowchart. PTA pure tone average, WRS word recognition score, THI Tinnitus Handicap Inventory

guidelines for clinical trials. The protocol is written in line with the Standard Protocol Items: Recommendations for Interventional Trials (SPIRIT) checklist (Additional file 1), as shown in Fig. 2.

\section{Recruitment}

Participants who are diagnosed with ISSNHL but did not respond to initial systemic treatment for at least 14 days are recruited by posters in the West China Hospital of Sichuan University.

\section{Participants}

Inclusion criteria

The inclusion criteria are:

1. Signed informed consent form; participants must be willing and able to provide consent for participation

2. Diagnosis of unilateral ISSNHL, defined as onset within $72 \mathrm{~h}$ affecting three consecutive frequencies of unknown aetiology [1]

3. Hearing loss occuring for at least 14 days but less than or equal to 1 year 


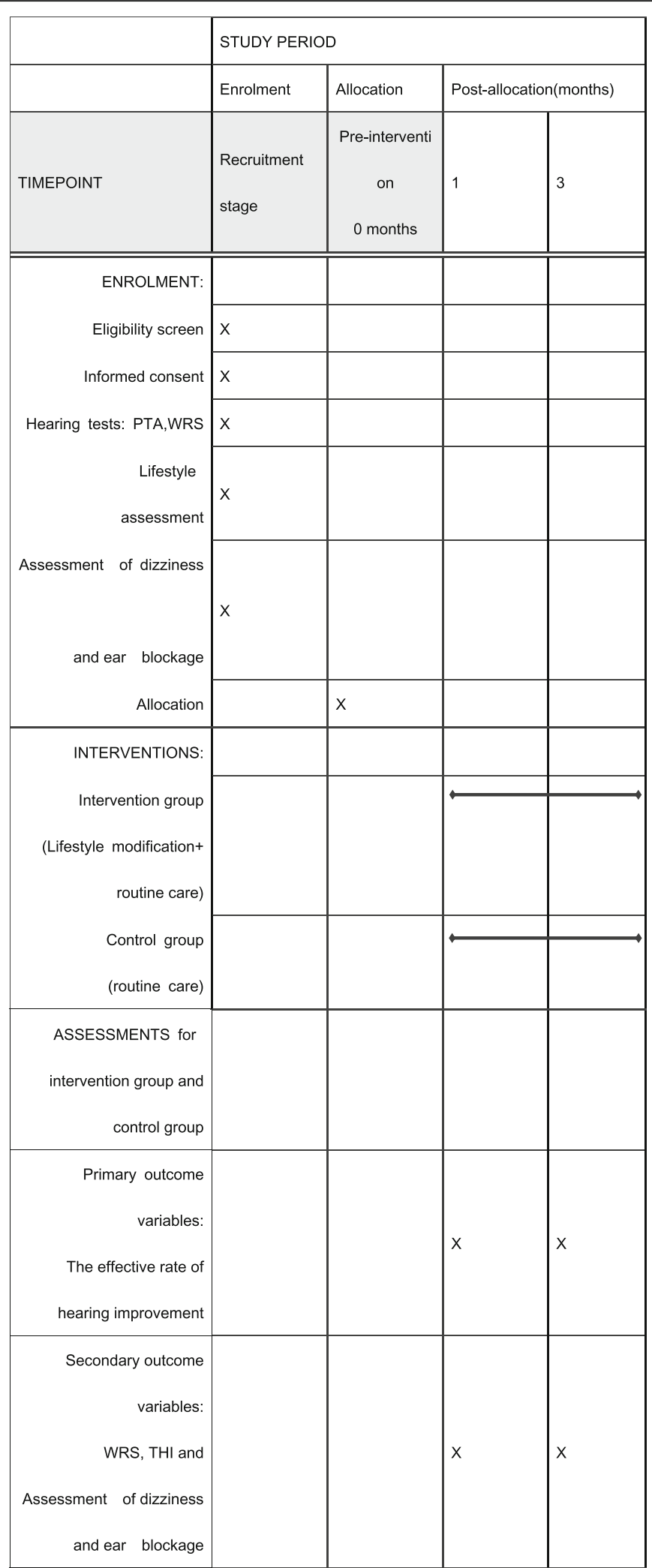

Fig. 2 SPIRIT figure: proposed schedule for enrolment, intervention and assessment. PTA pure tone average, WRS word recognition score, THI Tinnitus Handicap Inventory 
4. Insufficient recovery from ISSNHL for at least 14 days after onset and after receiving the Chinese ISSNHL guideline-recommended standard therapy

5. Reluctance to receive drugs, including steroid therapy

6. Patient age more than 18 years old but less than 60 years old

7. Hearing loss in the contralateral ear of at least 20 $\mathrm{dB}$

8. Patients who stopped receiving medication for more than 3 days.

\section{Exclusion criteria}

The exclusion criteria are as follows:

1. Previous disease or surgery in the affected ear

2. Hearing loss from an identified aetiology, including head trauma, conductive hearing loss, Meniere's disease or tumour

3. Inability to complete relevant assessments, such as cognitive impairment and mental disorder assessments

4. Serious comorbid conditions, such as progressive central disorder or life-threatening conditions

5. Any reason, in the investigator's opinion, that prohibits inclusion.

The criteria for trial termination and dropout are as follows: the patient develops a severe disease unrelated to participation in the trial; the patient chooses other treatments and drugs; the patient requests termination or withdraws; the patient no longer receives the trial treatment regimen and examination.

\section{Randomisation}

When patients want to participate in the trial and meet the requirements, a research assistant who reviews and explains the study collects the patient's basic demographic information and previous clinical data. Eligibility of the patient is ascertained, followed by provision of the written consent form. Before randomisation, the patients must have reassessed their hearing status with an otoscopic examination, hearing threshold tests (air conduction hearing thresholds measured at $250-8000 \mathrm{~Hz}$, bone conduction hearing thresholds measured at 500-4000 $\mathrm{Hz}$ ), word recognition scores (WRSs) and ear-specific immittance measurements (including tympanometry, static immittance and acoustic reflex measures). These measures can assess the patient's baseline hearing state and exclude conductive hearing loss. According to the classification standard of hearing loss degree published by the World Health Organization in 1997, the average values of hearing thresholds of 500, 1000, 2000 and $4000 \mathrm{~Hz}$ were calculated and then divided into categories of mild (26-40 dB hearing loss [HL]), moderate (41-60 $\mathrm{dB} H \mathrm{HL})$, severe $(61-80 \mathrm{~dB} \mathrm{HL})$ and profound $(\geq 81 \mathrm{~dB}$ $\mathrm{HL}$ ). Patients were stratified by gender, age and degree of hearing loss. Patients of the same gender, with age differences within 3 years and with the same degree of hearing loss were classified in the same stratification.

A statistician who is not part of the clinical intervention uses the Statistical Package for the Social Sciences (SPSS) 21.0 (IBM, Chicago, IL, USA) to generate a randomisation code. This code is embedded into serially numbered, opaque envelopes. After a participant completes the baseline measures, and when the same stratification reaches two or an even number of patients, another research assistant opens the next envelope in the series to determine the participant group allocation. Patients in the same stratification are randomised for the two treatments with a 1:1 ratio.

\section{Intervention}

Participants in the control group receive routine care, whereas those in the intervention group receive additional lifestyle counselling based on TCM. In this system, patient care focusses on health maintenance and prevention by encouraging patients to adhere to simple health and lifestyle practices [18].

Routine care includes two aspects, as follows:

- Educating participants about the natural history of ISSNHL and the limitations of existing evidence regarding efficacy; answering patients' questions about ISSNHL

- Counselling participants about the benefits of amplification, hearing-assistive technology and other supportive measures, especially for those whose hearing loss has lasted more than 3 months.

Lifestyle counselling consists of four sessions. The first step is the completion of the lifestyle survey of each participant and one-to-one targeted counselling based on the survey results.

- Diet. According to the theory of the Yellow Emperor's Inner Canon (Huang Di Nei Jing), a classical Chinese medicine book [19], yang qi is an important reason for maintaining normal human function. In addition, a food's energy can have a remarkable effect on health. Therefore, the diet should be dominated by staple foods, whereas 'cold' energy foods should be avoided. In simple terms, the central components of the dietary strategy are staple Chinese foods (with 'neutral' energy), such as rice and wheat. According to TCM principles, 'cold' 
energy foods include most fruits. The diet adopted in this study encourages participants to consume staple Chinese foods.

- Sleep. Patients sleep at night, avoid staying up late and wake up at dawn. The recommended time range for the patients to fall asleep is 10 p.m. to 11 p.m., and they need to be up by 5 a.m. to 7 a.m., thereby ensuring a sleeping window between $10 \mathrm{p} . \mathrm{m}$. and 5 a.m. Thus, reducing water intake prior to sleep is necessary to avoid waking up at night to urinate. Participants should not sleep during the day. However, a short nap of less than 30 min before 2 p.m. is advised for nonadaptive patients.

- Mood. A physician communicates with the participants to address their doubts and discuss the relationship between mood and ISSNHL and the importance of good mood to health. Participants' fear, despair and anxiety should be minimal.

- Physical activity. All participants are encouraged to be moderately physically active by doing taiji (a traditional Chinese sport) and housework and by walking and participating in leisure activities. Patients are discouraged from engaging in deliberate strenuous physical exercise, especially before going to bed. They are also discouraged to eat too much before going to bed.

The following measures are taken to improve patient compliance and reduce the dropout rate. All participants are entitled to free assessments, including audiology tests, one-to-one consultation and lifestyle assessment. In addition, no registration fee is required for the firstlevel expert outpatient service from the West China Hospital. At the end of the experiment, a free online consultation service is provided for 1 year.

Weekly one-on-one consultations and periodic checkups are provided over the phone, especially with the lifestyle modification group, to reinforce the importance of lifestyle change and answer related questions. Participants are encouraged to keep a symptom log on earassociated and systemic symptoms. The patients need to provide daily email updates regarding their lifestyle journal, including information on sleep and wake times and daily diet (Additional file 2). Participants are required to fill in the form daily for 1 month.

\section{Outcome measure evaluation}

The outcomes are evaluated at baseline and at 1 month and 3 months after the participant starts the intervention.

\section{Primary outcome measure}

The primary outcome measure will be the effectiveness of hearing improvement. This will be taken as the percentage of patients with an improvement of at least $15 \mathrm{~dB}$ in their impaired frequency compared with the baseline. On the basis of the American Academy of Otolaryngology-Head and Neck Surgery 2012 practice guideline on ISSNHL, an improvement within $10 \mathrm{~dB} \mathrm{HL}$ of initial HL or within $10 \mathrm{~dB}$ HL of the unaffected ear's hearing threshold is defined as complete recovery. An improvement of more than $30 \mathrm{~dB} \mathrm{HL}$ in pure tone average (PTA) from pretreatment hearing levels is defined as significant recovery; an improvement of $15-30 \mathrm{~dB} H \mathrm{HL}$ is defined as effective recovery. An improvement of less than $15 \mathrm{~dB}$ HL in PTA is defined as no recovery $[1,20]$.

\section{Secondary outcome measures}

Based on the visual analogue scale, the secondary outcomes include improvement of adherence to TCM lifestyle and evaluation of changes in WRS [21], Tinnitus Handicap Inventory (THI) [22] for patients with tinnitus and change in common symptoms, such as dizziness and ear blockage. These outcomes are measured at the first visit and during protocol visits.

\section{Blinding}

The audiologist, research assistants and statisticians involved in the study are blinded to the allocations. Given the nature of the counselling, blinding amongst consultants and patients is impossible. Thus, consultants and other researchers do not communicate amongst one another about the patient group during the trial. Patients also keep their treatment methods confidential. At the completion of the trial, patients in the control group are offered access to the lifestyle modification intervention.

\section{Sample size}

To the best of our knowledge, no randomised pilot study has been conducted to assess the effectiveness of lifestyle changes on ISSNHL. Therefore, we are not able to calculate the sample size based on previous studies. On the basis of our retrospective analysis (unpublished) and clinical experience, the efficiency ratio of the intervention group is conservatively estimated to be $50 \%$, and the natural recovery rate over 2 weeks is $10 \%$ [9]. Using a formula [23] to calculate the sample size of optimal treatment in the clinical trial and considering $\alpha=0.05$, $\beta=0.1$, by the table of normal distribution quantifiers $U_{\alpha(0.05)}=1.65, U_{\beta(0.1)}=1.28$, we require a patient sample size of 23 per group. We allow for a $20 \%$ loss to followup (approximately 10 cases), with a total sample size of 56 patients (28 per group) in the study.

$$
\begin{aligned}
& n=(U \alpha+U \beta)^{2} 2 P(1-P) /\left(P_{1}-P_{0}\right) 2, \\
& P=\left(\mathrm{P}_{1}+\mathrm{P}_{0}\right) / 2 \times 100 \%,
\end{aligned}
$$

$P_{0}$ : original efficacy, $P_{1}$ : expected efficacy. 


\section{Safety}

Any adverse events or discomfort throughout the course of the trial will be recorded by patients and data collectors. Participants may withdraw from the study for any reason at any time. The researchers will record the reasons on case report forms.

\section{Statistical analysis}

Data are analysed using SPSS V.21.0 (Chicago, IL, USA), with the significance level set at 0.05 (two-tailed) by statisticians who are independent of the research team. Patient baseline characteristics are summarised by treatment arm by employing appropriate summary statistics to assess baseline comparability only. Data analysis is conducted with the intention-to-treat (ITT) principle and a per-protocol (PP) analysis. To ensure the comparability of baseline conditions between the two groups and allow the presence of noncompliant patients, the ITT population consists of all randomised participants. In addition, at least one follow-up will be recorded after the intervention. According to the patients' actual lifestyle adjustment after random grouping, the PP analysis studies the patients who completed the trial and did not violate the protocol. This process is an explanatory, supplementary analysis, contributing to study objectivity. We calculate the effective rates at 1 and 3 months for the primary outcome and compare the intervention and control groups by using the $x^{2}$ test. For secondary outcomes, continuous variables, including THI, visual analogue scale and WRS, are compared between the two groups at all follow-up time points by using a $t$ test or the Wilcoxon signed-rank test as appropriate. Categorical variables, such as different degrees of hearing loss, are compared using the $\chi^{2}$ test or Fisher's exact test. For dropout analysis, we use multiple imputations for ITT analysis. A sensitivity analysis is performed to assess the effect of missing data assumptions.

\section{Data management}

Data accuracy is ensured by completing the paper copies of the case report form. Two independent researchers blinded to the group allocation input the data on an Excel spreadsheet, and the data are checked twice. Data are validated using original case report forms when any discrepancy is discovered. Paper files and electronic documents are stored separately in a locked filing cabinet and on a protected computer. Only the principal researchers are allowed access to the data. Researchers are unable to modify the data. They shall keep the information strictly confidential and shall not disclose it under any circumstances. The researchers shall sign a confidentiality agreement.

\section{Discussion}

ISSNHL, for which the aetiology remains unknown, is an acute disorder that occurs throughout life. Although $49-89 \%$ of patients achieve normal hearing through existing therapy with oral or intravenous steroids and with 32-65\% spontaneous recovery rates, treatment amongst patients who have incomplete recovery from ISSNHL after failure of initial management remains a problem [8-10]. Some patients are reluctant to receive recommended glucocorticoid treatment because of concerns regarding side effects, contraindications and drugto-drug interactions. The salvage therapy recommended by the 2012 ISSNHL guidelines is steroid perfusion, which results in hearing improvement ranging from 53 to $90 \%$ in the treatment group $[24,25]$. The dose and concentration of steroids vary similarly to the criteria used to define hearing improvement.

Previous studies indicated that patients who do not show any improvement within the first 14 days are unlikely to show remarkable recovery afterwards $[9,10]$, and thus they usually lose hope and discontinue therapy. Therefore, an acceptable and simple therapy is required to improve the effects of refractory ISSNHL for these patients.

TCM has been used for thousands of years and is widely accepted for treating diseases in China. Lifestyle, as part of TCM, has been integrated into the Chinese culture. Tradition and sustained interest in the benefits of a TCMrecommended lifestyle have remained, especially for patients whose conditions have not been effectively treated by Western medicine. We designed this trial to verify that a TCM-based lifestyle can provide help concerning hearing loss and concomitant symptoms amongst patients with ISSNHL. If successful, this intervention may help patients with refractory ISSNHL in China.

The study is designed to explore the efficacy of a TCM-based lifestyle change for patients with ISSNHL who have no or insufficient recovery after initial systematic Western medicine treatment. Although intratympanic steroid perfusion for this kind of ISSNHL has been used in Western treatment, the evidence of its efficacy remains unclear. In this study, the effective rate of hearing improvement is used as the primary outcome measure and is the most common parameter for ISSNHL. Secondary outcome measures include WRS, THI and accompanying symptoms, including dizziness and fullness of the ear. In addition to hearing loss, many people with ISSNHL complain of tinnitus and dizziness. The exact mechanisms that explain the effects of a TCM-based lifestyle change on ISSNHL require further detailed research and discussion in the future.

\section{Study limitations}

One of the major drawbacks of this study is that the currently widely adopted lifestyle questionnaire is not used. 
Lifestyle includes sleep, diet, mood and exercise. Thus, some factors that play a key role in improving ISSNHL remain unknown. Therefore, well-designed and randomised controlled trials that compare different lifestyle factors with one another are necessary in the future.

\section{Trial status}

The trial is currently recruiting patients. We have completed patient recruitment in October 2019.

\section{Supplementary information}

Supplementary information accompanies this paper at https://doi.org/10. 1186/s13063-019-3733-5.

Additional file 1. SPIRIT checklist: recommended items to address in a clinical trial protocol and related documents.

Additional file 2. Lifestyle diary. Participant completes the table to record a journal of sleep time and daily diet.

\section{Abbreviations}

ISSNHL: Idiopathic sudden sensorineural hearing loss; PTA: Pure tone average; TCM: Traditional Chinese medicine; THI: Tinnitus Handicap Inventory; WRS: Word recognition score

\section{Acknowledgements}

The authors acknowledge Professor Meng Hongdao from the University of South Florida for his encouragement in the preparation of the protocol.

\section{Authors' contributions}

DL designed this trial. YPF drafted the manuscript. YZ and PL contributed to supervise this study and participated in revising the manuscript. PZ and JZL provided advice and support. GL was responsible for the statistical analysis. All authors read and approved the final manuscript.

\section{Funding}

There is no funding for this protocol. This trial will be conducted with no external funding; it will instead be funded from the education fund for graduate students and Professor Zheng's individual research fund.

\section{Availability of data and materials}

The datasets used and/or analysed during the current study are available from the corresponding author on reasonable request.

\section{Ethics approval and consent to participate}

This trial has been approved by the Biomedical Branch of the Ethics Committee of the West China Hospital of Sichuan University (identifier 2016180). Study participation is voluntary and can be cancelled at any time without provision of reasons and without negative consequences for patients' future medical care. Informed consent will be obtained from all study participants before they enrol in the trial.

\section{Consent for publication}

All authors and investigators/patients give their consent for publication.

\section{Competing interests}

The authors declare that they have no competing interests.

\section{Author details}

${ }^{1}$ Hearing Center/Hearing and Speech Science Laboratory, Department of Otolaryngology Head and Neck Surgery, West China Hospital of Sichuan University, Chengdu, People's Republic of China. ${ }^{2}$ Department of Otolaryngology Head and Neck Surgery, The Affiliated Hospital of Southwest Medical University, 25 Taiping street, Jiangyang District, Luzhou, Sichuan 646000, People's Republic of China. ${ }^{3}$ Department of Otolaryngology Head and Neck Surgery, The First Affiliated Hospital of Guangzhou University of
Chinese Medicine, No. 16 Yard, Airport Road, Guangzhou 510405, People's Republic of China.

Received: 9 January 2019 Accepted: 17 September 2019

Published online: 02 December 2019

\section{References}

1. Stachler RJ, Chandrasekhar SS, Archer SM, et al. Clinical practice guideline: sudden hearing loss. Otolaryngol Head Neck Surg. 2012;146:S1-35

2. Nosrati-Zarenoe R, Arlinger S, Hultcrantz E. Idiopathic sudden sensorineural hearing loss: results drawn from the Swedish national database. Acta Otolaryngol. 2007;127:1168-75.

3. Teranishi M, Katayama N, Uchida Y, Tominaga M, Nakashima T. Thirty-year trends in sudden deafness from four nationwide epidemiological surveys in Japan. Acta Otolaryngol. 2007;127:1259-65.

4. Schreiber BE, Agrup C, Haskard DO, Luxon LM. Sudden sensorineural hearing loss. Lancet. 2010:375:1203-11.

5. Penido NO, Cruz OL, Zanoni A, Inoue DP. Classification and hearing evolution of patients with sudden sensorineural hearing loss. Braz J Med Biol Res. 2009;42:712-6.

6. Lazarini PR, Camargo AC. Idiopathic sudden sensorineural hearing loss: etiopathogenic aspects. Braz J Otorhinolaryngol. 2006;72:554-61.

7. Chau JK, Lin JR, Atashband S, Irvine RA, Westerberg BD. Systematic review of the evidence for the etiology of adult sudden sensorineural hearing loss. Laryngoscope. 2010;120:1011-21.

8. Moskowitz D, Lee KJ, Smith HW. Steroid use in idiopathic sudden sensorineural hearing loss. Laryngoscope. 1984;94:664-6.

9. Mattox DE, Simmons FB. Natural history of sudden sensorineural hearing loss. Ann Otol Rhinol Laryngol. 1977:86:463-80.

10. Wilson WR, Byl FM, Laird N. The efficacy of steroids in the treatment of idiopathic sudden hearing loss. A double-blind clinical study. Arch Otolaryngol. 1980;106:772-6.

11. Moon IS, Kim J, Lee SY, Choi HS, Lee WS. How long should the sudden hearing loss patients be followed after early steroid combination therapy? Eur Arch Otorhinolaryngol. 2009;266:1391-5

12. Plontke SK. Diagnostics and therapy of sudden hearing loss. GMS Curr Top Otorhinolaryngol Head Neck Surg. 2017;16:Doc05.

13. Lai D, Zhao F, Jalal N, Zheng Y. Intratympanic glucocorticosteroid therapy for idiopathic sudden hearing loss: meta-analysis of randomized controlled trials. Medicine. 2017:96:e8955

14. Sanchez-Munoz LA, Martin Merino M, Zalama Sanchez D. Diseaserelated malnutrition: a transversal improvement area. Med Clin. 2017; 149:89-90.

15. Sajjadi H, Paparella MM. Meniere's disease. Lancet. 2008;372:406-14.

16. Schultz AR, Neves-Souza RD, Costa Vde S, Meneses-Barriviera CL, Franco PP, Marchiori LL. Is there a possible association between dietary habits and benign paroxysmal positional vertigo in the elderly? The importance of diet and counseling. Int Arch Otorhinolaryngol. 2015;19:293-7.

17. Liu YQ, Chen ZJ, Li G, Lai D. Effects of educational counseling as solitary therapy for chronic primary tinnitus and related problems. Biomed Res Int. 2018:2018:1-9.

18. Unschuld PU, Tessenow H, Zheng J. Huang Di nei jing su wen: an annotated translation of Huang Di's Inner Classic - Basic questions (2 volumes). Oakland: University of California Press; 2011.

19. Ni M. The yellow emperor's classic of medicine: a new translation of the neijing suwen with commentary. Boulder: Shambhala Publications; 2015 .

20. Editorial Board of Chinese Journal of Otorhinolaryngology Head and Neck Surgery, Society of Otorhinolaryngology Head and Neck Surgery, Chinese medical Association. Guideline of diagnosis and treatment of sudden deafness (2015). Zhonghua Er Bi Yan Hou Tou Jing Wai Ke Za Zhi. 2015;50: $443-7$ In Chinese.

21. Jan TA, Kozin ED, Kanumuri W, Sethi RK, Jung DH. Improvement in word recognition following treatment failure for sudden sensorineural hearing loss. World J Otorhinolaryngol Head Neck Surg. 2016;2:168-74.

22. Ding $X$, Zhang $X$, Huang $Z$. The characteristic and short-term prognosis of tinnitus associated with sudden sensorineural hearing loss. Neural Plasticity. 2018:2018:6059697.

23. Carlin JB, Doyle LW. Sample size. J Paediatrics Child Health. 2002;38:300 
24. Ho HG, Lin HC, Shu MT, Yang CC, Tsai HT. Effectiveness of intratympanic dexamethasone injection in sudden-deafness patients as salvage treatment. Laryngoscope. 2004;114:1184-9.

25. Xenellis J, Papadimitriou N, Nikolopoulos T, et al. Intratympanic steroid treatment in idiopathic sudden sensorineural hearing loss: a control study. Otolaryngol Head Neck Surg. 2006;134:940-5.

\section{Publisher's Note}

Springer Nature remains neutral with regard to jurisdictional claims in published maps and institutional affiliations.

Ready to submit your research? Choose BMC and benefit from:

- fast, convenient online submission

- thorough peer review by experienced researchers in your field

- rapid publication on acceptance

- support for research data, including large and complex data types

- gold Open Access which fosters wider collaboration and increased citations

- maximum visibility for your research: over $100 \mathrm{M}$ website views per year

At $\mathrm{BMC}$, research is always in progress.

Learn more biomedcentral.com/submissions 